\title{
Innovative sensing technologies for nuclear instrumentation
}

\author{
Pattrick Calderoni, David H Hurley, \\ Joshua E Daw, Austin D Fleming \\ May 2019
}

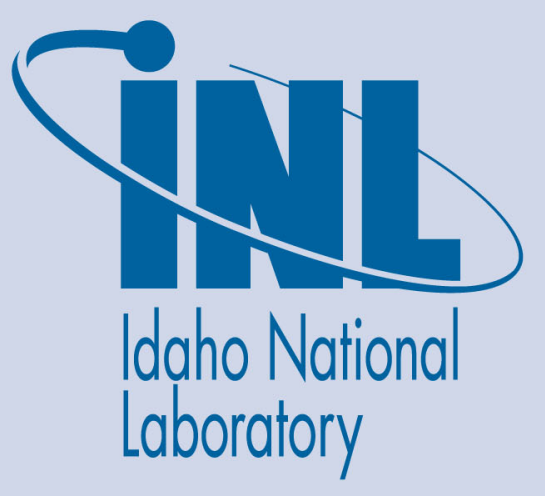

The INL is a U.S. Department of Energy National Laboratory operated by Battelle Energy Alliance 


\section{Innovative sensing technologies for nuclear instrumentation}

Pattrick Calderoni, David H Hurley, Joshua E Daw, Austin D Fleming

May 2019

Idaho National Laboratory Idaho Falls, Idaho 83415

http://www.inl.gov

Prepared for the

U.S. Department of Energy

Under DOE Idaho Operations Office

Contract DE-AC07-05ID14517 
Innovative sensing technologies for nuclear instrumentation

Pattrick Calderoni, David Hurley, Josh Daw, Austin Fleming

Idaho National Laboratory

PO Box 1625, Idaho Falls, ID, 83415

pattrick.calderoni@inl.org

\begin{abstract}
The Idaho National Laboratory (INL) is engaged in research and development activities for the development of nuclear instrumentation that contribute to advances in measurement methodologies, measurement systems, instrumentation, and sensors in the area of nuclear engineering. The activities described in this paper are centered on the INL Measurement Science Department, which was formed in 2018. Their mission is to develop instrumentation that provides real time, accurate, spatially resolved information regarding test conditions and the performance of fuels and materials during irradiation. This paper describes the integration of innovative optical fiber, acoustic and electrical impedance sensing technologies and measurement techniques as part of the activities.
\end{abstract}

Keywords-nuclear power, nuclear instrumentation, fiber optics, acoustic sensor

\section{INTRODUCTION}

Today, nuclear energy, utilizing Light Water Reactor (LWR) technology, provides about $20 \%$ of electricity production in the US and about $12 \%$ internationally. The U.S. nuclear fleet accounts for over $60 \%$ of all low-carbon electricity. Because reliable, grid-stabilizing, low emission electricity generation, energy security, and energy resource diversity will be increasingly valued, nuclear power's share of electricity production has a potential to grow domestically and internationally. In addition, a similar growth is expected for the application of nuclear energy for applications other than electricity generation, including process heat, desalination and hydrogen production.

As the US Department of Energy (DOE) nuclear laboratory, the Idaho National Laboratory (INL) is tasked with establishing the conditions for the growth of nuclear power thru research and development activities. One of the main focus is to accelerate the deployment of advanced nuclear technologies by develop, demonstrate and qualify advanced reactor fuel forms. However, advanced fuel and material development requires understanding irradiation effects on fuels and materials performance and relies on irradiation experiments ranging from tests aimed at targeted scientific questions to more integral effects underpin prototypic conditions. With the aim of developing a more fundamental understanding of fuels and materials performance, more specialized experiments and measurements are needed than the empirically-based approaches of the past. Today, to meet many of the aggressive goals in DOE's Nuclear Energy research portfolio and the need of its industrial partners, a new approach for in-pile instrumentation development is being implemented. This new approach leverages on the decade long experience in the design and fabrication of instrumentation for irradiation tests [1] and is centered on the INL Measurement Science Department, which was formed in 2018. Critical gaps in existing technology are addressed by an interdisciplinary approach that integrates three aspects: material science, advanced manufacturing, modeling and simulation. Materials science activities will be tasked with rapidly screening application specific sensor materials for high temperature and radiation environments. Advanced manufacturing activities will develop additive, micro, and nano manufacturing techniques that will allow a more efficient integration of sensors in irradiation test rigs and ultimately directly onto fuels and materials. Modeling and simulation activities will develop tools and capabilities to predict the behavior of sensors and sensor materials in pile. Critical outcomes include the following:

- Reduced development lifecycle for in-reactor instrumentation and subsequently the overall fuel system development cycle

- New technologies for in-pile characterization of materials behavior

- Sensors that employ multiple methodologies (multimode) that improve data quality

- Data at smaller length scales that provide insights into radiation-induced property evolution

- Connect changes in materials properties to changes in microstructure and chemistry

- Non-intrusive technologies

Individually, these advances are important; collectively, they should guarantee long-term revolutionary advances while continuing to leverage past and current activities for the evolution of existing technology.

\section{INNOVATIVE SENSORS TECHNOLOGIES}

The activities described in the paper are funded by two main DOE programs: In-Pile Instrumentation $\left(\mathrm{I}^{2}\right)$, and the development of instrumentation for the INL Transient Reactor Test Facility (TREAT). Together they provide a comprehensive approach to R\&D activities, which: (1) ensures the availability of a qualified set of baseline instrumentation, (2) complements it with the development of innovative sensors with disruptive potential in terms of performance and 
integrated measurement systems that address specific material science issues, (3) provides the capabilities and infrastructure to demonstrate and maintain the aforementioned technologies. This paper focuses on the second item, the integration of innovative sensing technologies in the development of nuclear instrumentation.

The need for innovative sensors to support nuclear fuel development derives from the complexity of the materials performance under irradiation and the constraints imposed by the deployment of instrumentation in the core of Material Test Reactors (MTR), where irradiation tests are performed. . Figure 1 presents the measurement needs within the propertystructure-modeling nexus for core materials. Measurements of temperature, pressure, and neutron flux are needed to characterize test conditions and can be used to directly validate modeling and simulation work. Measurement of materials properties will require the development of integrated measurement systems designed to characterize single effects, including thermal properties (thermal conductivity), mechanical properties (deformation in the elastic and creep regime, radial swelling, crack growth and void formation), chemistry (hydride formation and corrosion, crud build-up) and microstructure (recrystallization).

\section{OPTICAL FIBER SENSORS}

Measurement techniques based on optical fibers have been identified as a high risk, high reward component of the $\mathrm{I}^{2}$ program. Fiber optic sensors based on standard fused-silica have demonstrated the capability to provide multi-sensing (measuring different operational parameters within a single sensor configuration, such as temperature, pressure and strain) and multiplexing (communicating data collected at multiple locations through a single line) instrumentation. They are intrinsically immune to electromagnetic interference, electrically passive, compatible with a number of different sensing methodologies, and widely available at reasonable cost. In addition to the widespread use for telecommunication applications, instruments based on silica fibers are deployed in industrial applications at temperatures approaching 300-400 ${ }^{0} \mathrm{C}$ for applications such as distributed temperature sensing in oil and gas recovery. Ongoing research is testing the limits of amorphous silica as fiber material with respect to operational temperature. At temperatures above $500{ }^{\circ} \mathrm{C}$, conventional silica fibers can suffer from instabilities caused by interactions with various environmental species (especially hydrogen and/or water). While the application of protective coatings can alleviate environmental concerns, the amorphous structure of fused silica implies inherent instability when approaching the so-called 'annealing' temperature $\left(1000-1100{ }^{\circ} \mathrm{C}\right)$ at which the silica network will begin to relax internal strain (recrystallization). Successful utilization of silica fibers is rarely reported at temperatures higher than $900{ }^{\circ} \mathrm{C}$ due to the inherent thermal instability of the silica fiber substrate itself.

\section{A. Radiation resistant optical fiber materials}

The amorphous structure of fused silica is strongly impacted by radiation. Three mechanism are responsible at the macroscopic scale for the degradation of the fiber performance: Radiation Induced Attenuation (RIA), which increases light adsorption in the material (darkening); Radiation Induced Emission (RIE), which generates unwanted light (optical noise) and Radiation Induced Compaction (RIC), which causes variations in the density of the fiber material resulting in changes of optical properties. Various dopants such as fluorine, chlorine, hydrogen, $\mathrm{OH}$, have been tested to improve the defects caused by irradiation. The dopants improve RIA of the fiber at certain wavelength but worsens it at the other. For instance, Fluorine doped fibers perform better at infra-red wavelength, but has higher attenuation in the visible regime. Overall, pure silica and Fluorine doped silica have shown the highest promise at $1550 \mathrm{~nm}$ and are the reference material for optical fiber sensors under investigation for nuclear applications.

Sapphire $(\alpha-$ A12O3) fibers are recognized as hightemperature alternative to amorphous silica due to the high melting temperature (about $2054{ }^{\circ} \mathrm{C}$ ), outstanding chemical resistance and mechanical strength of their crystalline network. They have a wide transmission window, low optical losses and high damage threshold, making it an ideal candidate for multiple sensing techniques that can measure temperature, strain, deformation, pressure and chemical composition in extreme environments. However, as-fabricated sapphire fibers are inherently multi-mode, where the light is not confined to a single optical path, causing significant attenuation and making it impossible to use for most sensing techniques that are based on interferometric and distributed interrogation. To overcome this challenge a cladding must be formed by modifying the structure of the outer layer of the fiber core. The INL is collaborating with the Ohio State University to develop a process to create a cladding in pure sapphire fibers using ions generated by neutron capture in a lithium containing material. The ${ }_{3}^{6} \mathrm{Li}(\mathrm{n}, \alpha){ }_{1}^{3} \mathrm{H}$ reaction creates high energy alpha particles and tritons that interact with the fiber outer layer uniformly along its entire length to a depth of about 24 microns. The damage created by the ions in the sapphire network changes its optical properties (in particular, the index of refraction) enough to create a cladding similar to that obtained by doping the outer layer of fused silica fibers. The end result is a sapphire fiber with single or highly reduced modes transmission and minimal attenuation losses.

\section{B. Radiation resistant optical fiber sensors and measurement techniques}

The near term application of optical fibers to in-pile instrumentation is to enable Infrared (IR) pyrometry. In this application optical fibers are used simply as light guide, allowing real time, remote characterization of the light emitted by nuclear fuel cladding surfaces during operation. IR pyrometry calculates temperature by analyzing the measured blackbody emission from a specimen according to Planck's law. This technique has the ability to perform high-speed, hightemperature, non-contact temperature measurements, which makes it highly desirable for in-pile transient testing in TREAT. The first deployment for the IR pyrometer in a TREAT experiment is in the ATF-SETH experiment series. This test series consists of 4 dry atmosphere experiments with varying pulse sizes, which will result in four different target temperatures of $1300 \mathrm{~K}, 2300 \mathrm{~K}, 2900 \mathrm{~K}$, and fuel melt 
$(\sim 3100 \mathrm{~K})$. The instrumentation consists of four thermocouples attached to the fuel cladding at different axial locations, and two optical lines used for pyrometry measurements. Before the assembly of the ATF-SETH capsules a mock-up, without fuel in the rodlet, was performed to practice all steps of the assembly. A photo of this mock-up assembly can be seen in Figure 2. . The fiber selected for this application has been commercially acquired (fiberguide AFS600/660/710T), and is based on $\mathrm{F}$ dopes amorphous silica. It has a $710 \mu \mathrm{m}$ diameter with a polyimide coating (for low temperature test), and 660 $\mu \mathrm{m}$ without the coating. Conax (C) Multi-Hole Ceramic (MHC) feedthroughs with Grafoil seals are used either directly on coated fibers or using stainless steel sheathing for bare fibers (Figure 3).

Beyond the use as light guide, several types of optical sensors and related measurement techniques have been considered for nuclear applications. In order of increasing complexity in terms of development, they include: extrinsic sensors based on Fabry-Perot interferometry; Fiber Bragg Gratings (FBG); distributed optical sensing techniques (Rayleigh, Brillouin, Raman), or more specifically, Optical Backscattering Reflectometry (OBR). Current activities focus on FBG sensors due to their maturity in other industrial applications, demonstrated potential for high temperature operation, multiplexing capability and ease of deployment (intrinsic sensors). A fiber Bragg grating (FBG) is a periodic modulation of the refractive index along the fiber length created traditionally by exposing the core of a photosensitive optical fiber to an intense ultraviolet light interference pattern. Radiation effect on FBG structures and on Bragg peak has been widely studied. The defects generated in the FBG manifest in two predominant responses - Radiation-Induced Attenuation (RIA) and Radiation-Induced Bragg Wavelength Shift (RI- BWS). RIA degrades the grating performance by decreasing the fiber transmission and amplitude of the reflected peak. RI-BWS shifts the entire peak (with linear dependence on the irradiation temperature) and changes its shape, affecting the quality of the FBG temperature sensor measurements. In addition, the Bragg peak is affected from radiation-induced dilation (negative compaction) changes due to the Radiation Induced Compaction (RIC) described earlier as part of fiber materials response. Because of these processes, traditional UV laser written FBG has shown to darken and disappear under irradiation and high temperature. However, in recent years, infrared (IR) laser based femtosecond-etched (fs) Bragg gratings have shown great promise for use in a nuclear reactor environment. The combined use of radiation resistant materials (to reduce the effect of RIA on long distance transmission) and fs FBGs offers a path to their application in core, and is the focus of the activities of INL and its collaborators. Recent results from deployment of sensors developed in collaboration with CEA and University of Pittsburgh in the Massachusetts Institute of Technology (MIT) research Reactor (MITR) are presented at this Conference in an accompanying paper.

\section{ACOUSTIC SENSORS}

The application of acoustic sensor and measurement techniques to in-pile instrumentation offer advantages complementary to optical fibers and a similar path towards demonstration and deployment. At first it should be clarified that the scope of the activities here described is currently limited to the measurement of irradiation test conditions, and the performance of fuel and materials in MTRs irradiation experiments. The application of acoustic measurements to Non Destructive Examination (NDE) of nuclear power plant components (ie, heat exchangers) or coolant loop operation (ie, mass flow meters) is not part of the scope, although it is expected that results will be leveraged to overcome common challenges.

\section{A. Ultrasound Thermometer}

The near term application of acoustic techniques is the Ultrasound Thermometer (UT), which has been under development at INL since 2015. Waveguide ultrasonic thermometry is based on measuring the time-of-flight of acoustic pulses propagated along a thin wire or rod. Narrow ultrasonic pulses are generated in a magnetostrictive rod by a short duration magnetic field pulse produced by an excitation coil (Figure 4). The ultrasonic pulse propagates to the waveguide, where a fraction of the pulse energy is reflected at each discontinuity (notches or diameter change). Each reflected pulse is received by the excitation coil, transformed into an electrical signal, amplified and evaluated in a start/stop counter system. The time interval between two adjacent echoes is evaluated and compared to a calibration curve to give the average temperature in the corresponding sensor segment. The multi-segment design of the thermometer allows temperature measurement of the individual segments via the time-of-flight between pulses reflected from the features (i.e. notches) that define these segments. Signal processing consists of filtering/averaging to improve signal to noise ratio, and tracking of waveform features (usually peak or zero-crossing detection) for delay time measurement.

The use of magnetostrictive materials (Remendur and Galfenol) as basis for the transducer design is motivated by their proven stability under irradiation. However, the identification of a radiation tolerant piezoelectric material would overcome some of the reliability issues encountered with the current UT design configuration. Aluminum nitride (AIN) has shown potential for high radiation tolerance as well as high temperature compatibility, with a Curie temperature over $2000 \mathrm{C}$ (possibly corresponding to its melting point of $2200 \mathrm{C}$ ). As such, AlN shows potential for use in-core as an ultrasonic transducer (applied directly as the waveguide material) and for several types of intrinsic sensors such as Surface Acoustic Wave (SAW) sensors to monitor temperature, gas pressure and composition.

\section{B. Laser-based Resonant Ultrasound Spectroscopy (RUSL)}

The development of an in-pile instrument designed to characterize the microstructure evolution of nuclear materials is the first example of the integrated measurement for single effect test introduced in Section II. This instrument combines acoustic and optical techniques, and similarly to the IR pyrometer relies on optical fibers to transmit light to a remote detector. The RUSL instrument uses laser-based resonant ultrasound to monitor grain restructuring during irradiation 
tests by relating changes in polycrystalline elastic properties to grain microstructure. The instrument performs repeated measurement of the resonant frequency of a vibrating beam fabricated from the material of interest. Light is used for excitation and detection of the beam vibrations which allows for transmission via two optical fibers (Figure 5). Excitation is accomplished using an amplitude modulated laser driven by a network analyzer. The laser light is coupled into an optical fiber and delivered to the base of the beam. Excitation occurs through optical heating and the resulting thermal expansion. The modulation frequency is swept over a range including the resonant mode of interest. Detection of the beam deflection is based on a fiber optic lever technique. Detection light is provided by a broad band source which is coupled into an optical fiber, passes through a bi-directional beam splitter and is then delivered to the tip of the beam. Light exiting the optical fiber is reflected from the sample surface and coupled back into the fiber where it propagates back toward the source. The intensity of the light returning to the core of the optical fiber is dependent on the distance between the fiber tip and the sample. Thus, the fluctuation of the tip of the cantilever beam causes an intensity modulation of the light propagating back toward the source, which is measured by a photoreciever. This detection method is independence of absolute light intensity which minimizes the effects of radiation induced attenuation in the optical fiber.

\section{ELECTRICAL SENSORS}

Electrical impedance based sensing is a mature measurement field with broad applications and commercially available instruments available for standard measurements. However, it has limited applications in in-pile monitoring because of challenging environmental conditions (high temperature and neutron irradiation) and design constraints (pressure vessel penetration and grounding issues). An example of a near term application of electrical sensors is the impedance based diameter gauge, which overcomes the design integration challenges and intrusiveness associated with the use of Linear Variable Differential Transformer (LVDT) devices that are normally deployed in irradiation tests. The INL has performed a proof of principle validation of the concept using a simple, two coaxial electrodes geometry that is applicable to the measurement of a fuel pin swelling during irradiation. The main goal of the validation was to characterize the method sensitivity for a typical fuel pin dimension of $10 \mathrm{~mm}$. The experimental results have been compared to analytical solutions and simulations performed with the commercial software package COMSOL Multiphysics. The results show that the sensor has a diameter measurement resolution of $<1$ $\mu \mathrm{m}$ (compared to a $10 \mu \mathrm{m}$ target) and $<20 \mu \mathrm{m}$ error $(<0.2 \%$ error on diameter measurements). The measured capacitance is a minimum when rod and sensor are concentric, resulting in insignificant sensitivity to small offset distances when the sensor is well-aligned.

\section{CONCLUSION}

The INL has formed the Measurement Science Department to address the need of developing nuclear instrumentation in support of DOE Nuclear Energy (NE) programs and industrial vendors engaged in the expansion of nuclear power as part of the US energy portfolio. This paper describes research and development activities aimed at developing innovative sensing technologies for application in Material Test Reactors (MTR) irradiation experiments, which are a key component of the demonstration of nuclear fuel and materials performance in support of the modernization of the current fleet and the deployment of advanced nuclear reactors.

\section{REFERENCES}

Comprehensive list of references will be provided with final manuscript submission.

[1] J. L. Rempe, D. L. Knudson, J. E. Daw, T. Unruh, B.M. Chase, J. Palmer, K. G. Condie, K. Davis, "Enhanced in-pile instrumentation at the Advanced Test Reactor", IEEE Trans. Nuc. Sc., Vol. 59, No. 4, pp. 1215-1223, Aug. 2012

[2] T. Unruh, B. Chase, J. Rempe, D. Nigg, G. Imel, J. Harris, T. Sherman, J.F. Villard, "In-core flux sensor evaluations at the ATR Critical facility” Nuc. Tech., Vol. 187, pp. 308-315, Sep. 2014

[3] J. L. Rempe, D. L. Knudson, K. G. Condie, S. Curtis Wilkins, "High Temperature Thermocouple Design and Fabrication", US Patent 2008/0205483 A1, 2008

[4] J. Daw, B. Tittmann, B. Reinhardt, G.Kohse, P. Ramuhalli, R. Montgomery, H.-T. Chien, J.-F. Villard, J. Palmer, J. Rempe, "Irradiation testing of Ultrasonic Transducers" IEEE Trans. Nuc. Sc., Vol. 61, No. 4, pp. 2279-2284, Aug. 2014

[5] D.L. Knudson, J. L. Rempe, "Linear variable differential transformer (LVDT)-based elongation measurements in Advanced Test Reactor high temperature irradiation testing" Meas. Sci. Technol., Vol. 23, pp. 1-7, 2012 
Figures will be selected and placed within text and formatted for final manuscript submission in order to meet the 6 page requirement.

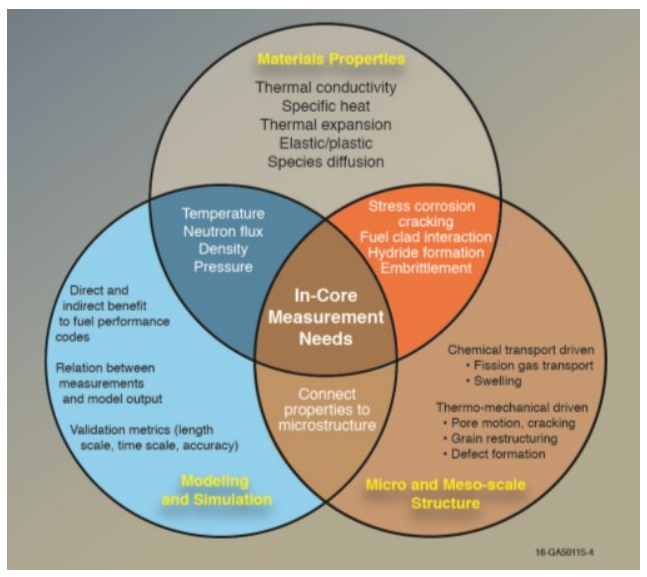

Fig. 1. In core measurement needs: the nexus between properties, modeling, and structure.

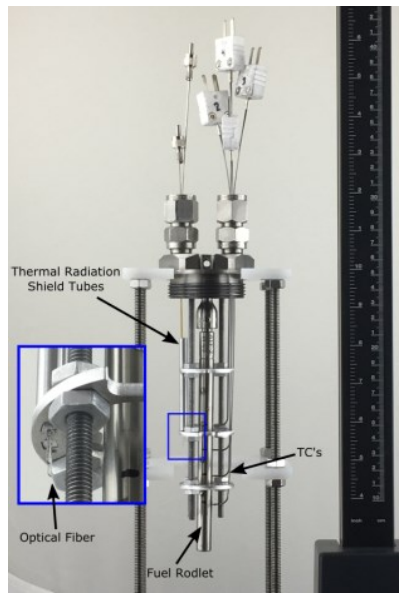

Figure 2. Mock-up of the SETH-ATF capsule lid with instrumentation installed

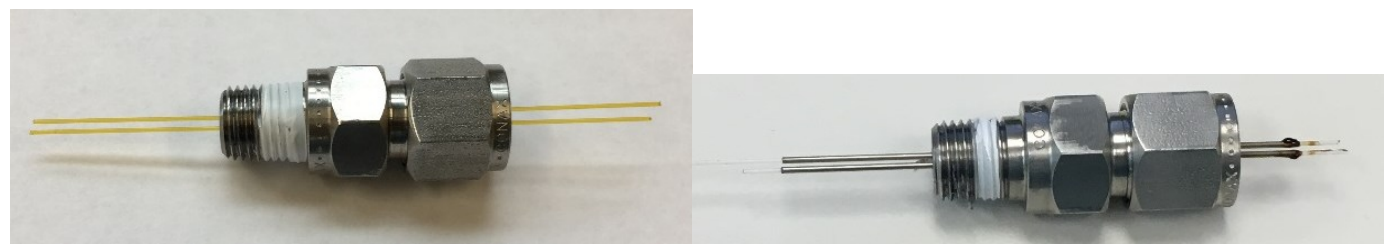

a) Coated fiber

b) Bare fiber with stainless steel sheathing

Figure 3. Images of the four configuraitons of fiber optic feed throughs that were leak tested 


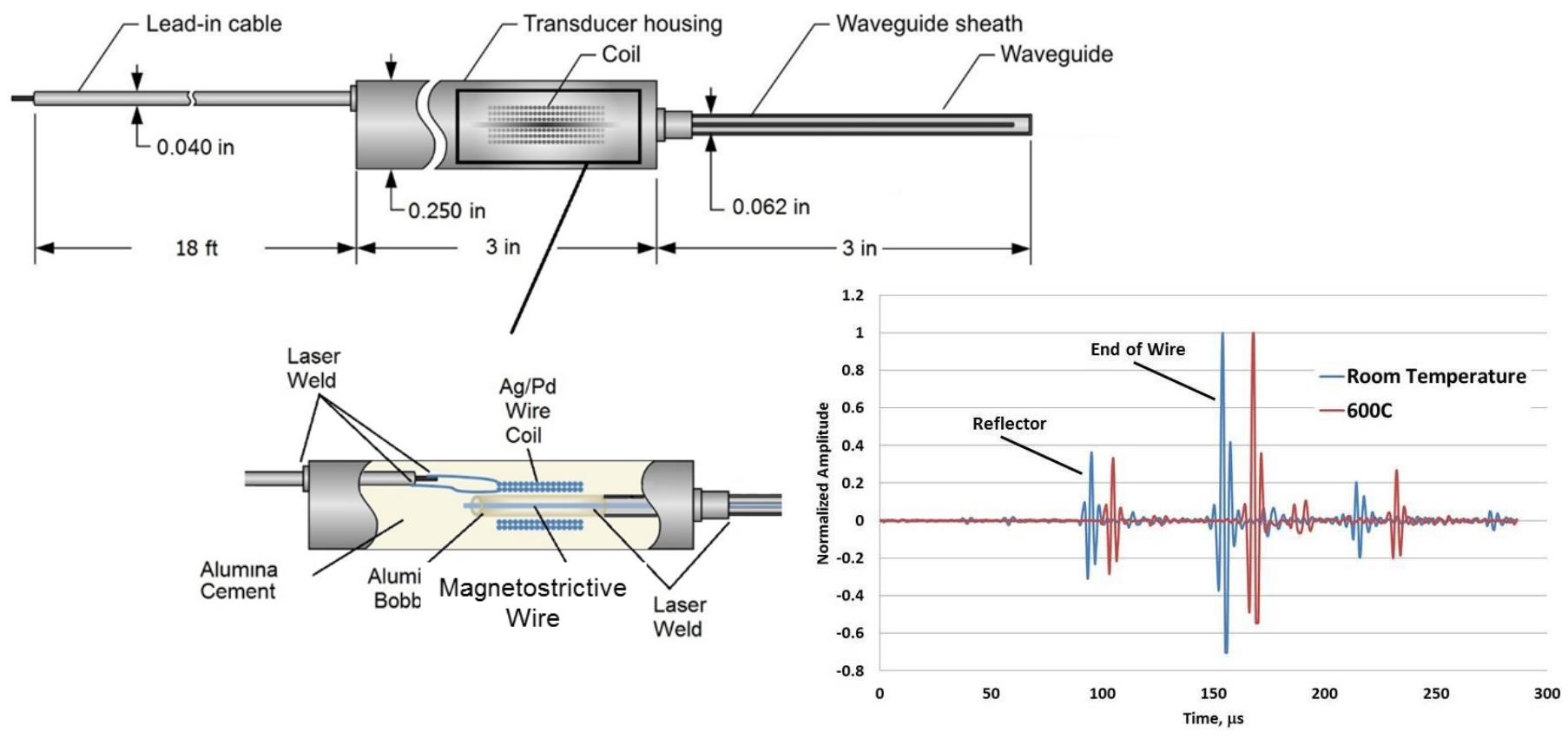

Figure 4. Magnetostrictive ultrasonic waveguide thermometer - design and results
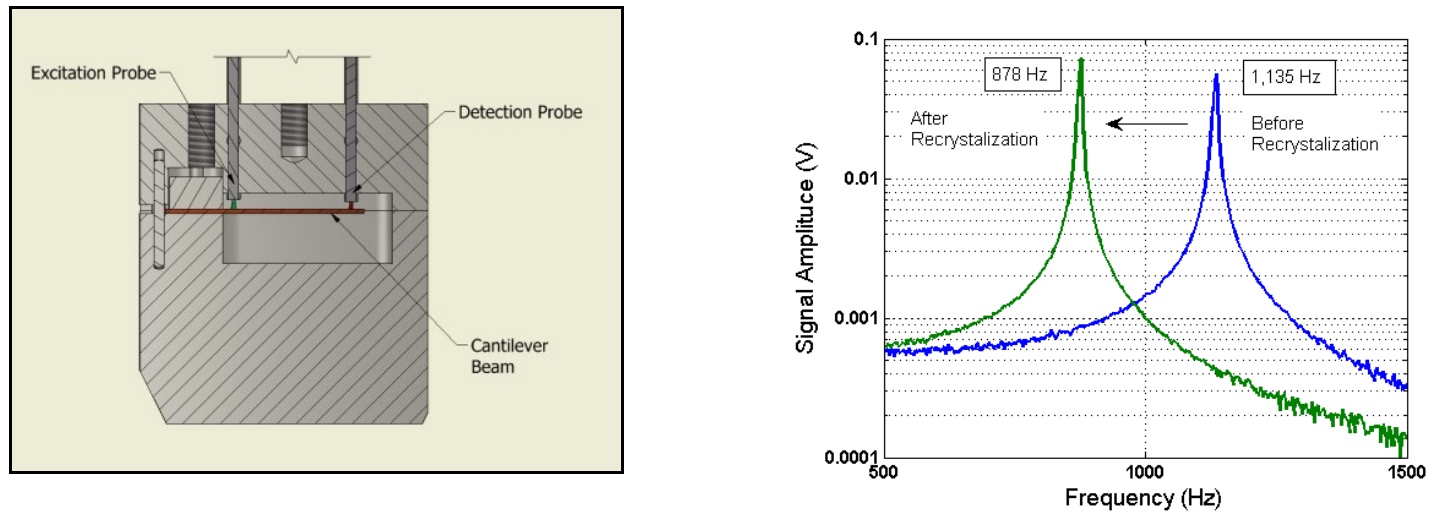

Figure 5. RUSL design and results
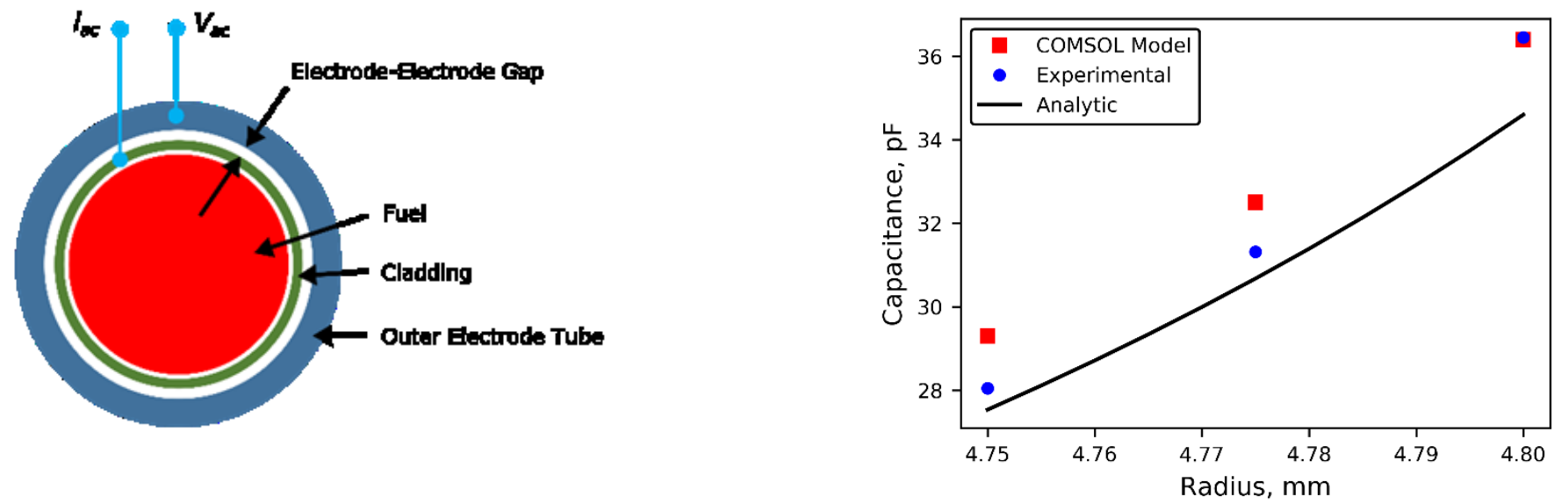

Figure 6. Impedance diameter gauge - design and results 